\title{
Sustainable inventory model with trade credit under carbon emission reduction investment
}

\author{
Juanjuan Qin
}

School of Business, Tianjin University of Finance and Economics, Tianjin 300222, China

Abstract: This paper discusses that the sustainable trade credit and inventory policy, with demand related to credit period and consumer environment sensitivity, under the carbon cap-and-trade regulation and tax regulation. And the firms make efforts to reduce the carbon emissions. The optimal credit period, order quantity and the carbon emission level of retailers can be obtained.

Keywords: inventory policy; trade credit; carbon emission reduction investment.

\section{Introduction}

Low-carbon economy has been deeply rooted in the hearts of the people, and become a consensus, along with the development of low carbon economy concept. Under this background, the supply chain operation began to increasingly consider low carbon factors. In the low carbon supply chain study, many researchers analyzed the emission reduction behavior and the effects of carbon emissions trading on supply chain decisions, such as Du et al. (2013), Qin et al.(2016). But these studies did not consider the impact of trade credit on decision making. In reality, the trade credit can stimulate the market demand (Gao et al., 2014). Therefore, in the paper we mainly integrate the trade credit contract in the research of low carbon supply chain.

\section{Problem definition and notation}

In the model, the demand rate is a positive exponential function of the credit period as $D(n)=\varepsilon e^{a n}$, where $\varepsilon$ and $a$ are positive constants. For convenience, $D(n)$ and $D$ will be used interchangeably. We derive the optimal production quantity, the credit period and the carbon emission reduction investment under the carbon emission cap and trade regulation. Considering a carbon emission intensive firm, it produces the product to meet the market demand under the carbon cap-and-trade regulation. We assume the unit price of the buying or selling are both equal to $\rho$.

Denote $C Q_{2}(Q)$ as the annual total carbon emission of the enterprise. For the given production quantity $Q$, the annual setup times are $D(n) / Q$ and the annual inventory holding cost is $h Q / 2$. The corresponding carbon emission linked to the setup times and the inventory holding are $D(n) \hat{A} / Q$ and $Q \hat{h} / 2$, respectively. The annual carbon emission during the production process is $\hat{c} D(n)$. Therefore, the total carbon emission related to producing, delivering and the storing product under the carbon emission reduction is defined as following:

$$
\mathrm{CO}_{2}(\mathrm{Q})=\hat{c} D(n)+\frac{D(n)}{Q} \hat{A}+\frac{Q}{2} \hat{h}-\xi D(n) .
$$

For the enterprise, the revenue is $s e^{-(j+k) n} D(n)$. And the cost related to the production process is $c D(n)+D(n) A / Q+Q h / 2$. Also, $\rho\left[Z-C Q_{2}(Q)\right]$ is the emission cost or revenue resulted from the buying or selling permits. When $Z-C Q_{2}(Q) \geq 0$, the enterprise carbon emissions are less than the carbon cap. Thus, the enterprise can sell the permit to obtain the revenue. Otherwise, when $Z-C Q_{2}(Q)<0$, the enterprise carbon emissions are more than the carbon cap. Thus, the enterprise can buy the 
permit to incur the cost. The investment of carbon emission reduction is $u \xi^{2} / 2$. Based on the rationale above, the annual profit of the enterprise $\Pi_{1}(n, Q, \xi)$ can be written as

$$
\Pi_{1}(n, Q, \xi)=s \mathrm{e}^{-(j+k) n} D(n)-c D(n)-\frac{D(n)}{Q} A-\frac{Q}{2} h-\frac{1}{2} u \xi^{2}+\rho\left[Z-\mathrm{CO}_{2}(\mathrm{Q})\right] .
$$

Eqs.(2) can be transformed into the following Eq.(3):

$$
\Pi_{1}(n, Q, \xi)=s \mathrm{e}^{-(j+k) n} D(n)-(c+\rho \widehat{c}) D(n)-\frac{D(n)}{Q}(A+\rho \widehat{A})-\frac{Q}{2}(h+\rho \widehat{h})-\frac{1}{2} u \xi^{2}+\rho \xi D(n)+\rho Z \cdot
$$

In what follows, we investigate and derive the enterprise's optimal production lot size, the optimal carbon reduction technology investment and then the optimal credit period.

\subsection{The optimal production lot size}

Maximizing the annual profit of the Eqs.(3) with respect to the production lot size, is equivalent to minimizing the following cost function:

$$
\operatorname{Min}_{Q}\left\{\frac{D(n)}{Q}(A+\rho \widehat{A})+\frac{Q}{2}(h+\rho \widehat{h})\right\}
$$

For simplicity, we use the arithmetic-geometric inequality method. It is easy to know that the optimal production lot size can be obtained when

$$
\frac{D(n)}{Q}(A+\hat{A} \rho)=\frac{Q}{2}(h+\hat{h} \rho) .
$$

Therefore, the optimal production lot size is

$$
Q_{1}^{2}=\frac{2 D(n)(A+\hat{A} \rho)}{(h+\hat{h} \rho)} .
$$

The corresponding optimal value of Eqs.(4) is

$$
\sqrt{2 D(n)(A+\hat{A} k)(h+\hat{h} k)} \text {. }
$$

\subsection{The optimal carbon reduction technology investments}

Maximizing the annual profit of the Eqs.(3) with respect to the carbon reduction technology investment, is equivalent to maximizing the following function:

$$
\operatorname{Max}_{\xi}\left[-\frac{1}{2} u \xi^{2}+\rho \xi D(n)\right] .
$$

It is easy to know that the optimal carbon reduction technology investment can be obtained when

$$
\xi_{1}^{*}=\rho D(n) / u \text {. }
$$

The corresponding optimal value of Eq.(8) is

\subsection{The optimal credit periods}

$$
\rho^{2} D^{2}(n) / 2 u \text {. }
$$

Based on the analysis of Section 3.1 and Section 3.2, the inventory problem of the enterprise is reduced to a single decision variable of $n$.

In this section, we try to obtain the optimal credit period for the enterprise. In order to find the optimal solution $n^{*}$, we derive the first and the second derivatives of $\Pi_{1}\left(n, Q^{*}, \xi^{*}\right)$ with respect to $n$ in the following.

$$
\begin{aligned}
& \frac{\partial \Pi_{1}\left(n, Q_{1}^{*}, \xi_{1}^{*}\right)}{\partial n}=s \varepsilon(a-j-k) \mathrm{e}^{(a-j-k) n}-(c+\rho \widehat{c}) \varepsilon a e^{a n}+\frac{\rho^{2} \varepsilon^{2} a e^{2 a n}}{u} ; \\
& -\frac{a\left(\varepsilon e^{a n}\right)^{1 / 2}}{2} \sqrt{2(A+\hat{A} \rho)(h+\hat{h} \rho)}
\end{aligned}
$$




$$
\begin{aligned}
& \frac{\partial^{2} \Pi_{1}\left(n, Q_{1}^{*}, \xi_{1}^{*}\right)}{\partial n^{2}}=s \varepsilon(a-j-k)^{2} \mathrm{e}^{(a-j-k) n}-(c+\rho \widehat{c}) \varepsilon a^{2} e^{a n}+\frac{2 \rho^{2} \varepsilon^{2} a^{2} e^{2 a n}}{u} . \\
& -\frac{a^{2}\left(\varepsilon e^{a n}\right)^{1 / 2}}{4} \sqrt{2(A+\hat{A} \rho)(h+\hat{h} \rho)}
\end{aligned} .
$$

Clearly, $\Pi_{1}\left(n, Q_{1}^{*}, \xi_{1}^{*}\right)$ is a continuous function of $n$ for $n \in[0, \infty)$. Therefore, $\Pi_{1}\left(n, Q_{1}^{*}, \xi_{1}^{*}\right)$ has a maximum value for $n \in[0, \infty)$. To identify whether $n$ is 0 or positive, we define the following discrimination term.

$$
\begin{aligned}
& \Delta_{1}=\left.\frac{\partial \Pi_{1}\left(n, Q_{1}^{*}, \xi_{1}^{*}\right)}{\partial n}\right|_{n=0}=s \varepsilon(a-j-k)-(c+\rho \widehat{c}) \varepsilon a+\rho^{2} \varepsilon^{2} a / u \\
& -a(\varepsilon)^{1 / 2} \sqrt{2(A+\hat{A} \rho)(h+\hat{h} \rho)} / 2
\end{aligned}
$$

If $\frac{\partial^{2} \Pi_{1}\left(n, Q_{1}^{*}, \xi_{1}^{*}\right)}{\partial n^{2}}<0, \Pi_{1}\left(n, Q_{1}^{*}, \xi_{1}^{*}\right)$ is strictly a concave function in $n$, hence exists a unique maximum solution $\tilde{n}_{1}$ obtained when $\frac{\partial \Pi_{1}\left(n, Q_{1}^{*}, \xi_{1}^{*}\right)}{\partial n}=0$. If $\Delta_{1} \leq 0$, then $\Pi_{1}\left(n, Q_{1}^{*}, \xi_{1}^{*}\right)$ is maximized at $n_{1}^{*}=0$; if $\Delta_{1}>0, \Pi_{1}\left(n, Q_{1}^{*}, \xi_{1}^{*}\right)$ is maximized with $n_{1}^{*}=\tilde{n}_{1}>0$. Based on Eqs.(12), it is easy to obtain if $s \varepsilon(a-j-k)^{2} \mathrm{e}^{(a-j-k) n} \leq(c+\rho \widehat{c}) \varepsilon a^{2} e^{a n}-2 \rho^{2} \varepsilon^{2} a^{2} e^{2 a n} / u, \Pi_{1}\left(n, Q_{1}^{*}, \xi_{1}^{*}\right)$ is strictly concave function in $n$. Hence, a unique maximum solution exists. If $\partial^{2} \Pi_{1}\left(n, Q_{1}^{*}, \xi_{1}^{*}\right) / \partial n^{2} \geq 0$, then $\Pi_{1}\left(n, Q_{1}^{*}, \xi_{1}^{*}\right)$ is a convex function of $n$. Therefore, the optimal solution of $\Pi_{1}\left(n, Q_{1}^{*}, \xi_{1}^{*}\right)$ is at one of the two boundary points ( 0 or $\left.\infty\right)$. Substituting $\infty$ with Eqs. (11), we have $\lim _{n \rightarrow \infty} \partial \Pi_{1}\left(n, Q_{1}^{*}, \xi_{1}^{*}\right) / \partial n<0$. Hence, $n=+\infty$ is not an optimal solution, which implies that the optimal solution is $n_{1}^{*}=0$. Consequently, the following results can be derived.

Theorem 1.if $s \varepsilon(a-j-k)^{2} \mathrm{e}^{(a-j-k) n} \leq(c+\rho \widehat{c}) \varepsilon a^{2} e^{a n}-2 \rho^{2} \varepsilon^{2} a^{2} e^{2 a n} / u$, then:

(1) $\Pi_{1}\left(n, Q_{1}^{*}, \xi_{1}^{*}\right)$ is strictly concave function in $n$, hence exists a unique optimal solution.

(2) If $\Delta_{1} \leq 0$, then $\Pi_{1}\left(n, Q_{1}^{*}, \xi_{1}^{*}\right)$ is maximized at $n_{1}^{*}=0$.

(3) If $\Delta_{1}>0$, then there exists a unique $\tilde{n}_{1}>0$ such that $\Pi_{1}\left(n, Q_{1}^{*}, \xi_{1}^{*}\right)$ is maximized at $n_{1}^{*}=\tilde{n}_{1}>0$.

\subsection{The global solutions}

Notice that the annual profit function of the enterprise $\prod_{1}(n, Q, \xi)$ has three decision variables with the production lot size, the carbon reduction technology investment and the credit period. Therefore, we need to prove the Hessian matrix is negative definite at $\left(n_{1}^{*}, Q_{1}^{*}, \xi_{1}^{*}\right)$ of $\Pi_{1}(n, Q, \xi)$. The Hessian matrix of $\Pi_{1}(n, Q, \xi)$ with respect to $(n, Q, \xi)$ is shown in the following.

$$
H_{1}=\left[\begin{array}{lll}
\frac{\partial^{2} \Pi_{1}(n, Q, \xi)}{\partial n^{2}} & \frac{\partial^{2} \Pi_{1}(n, Q, \xi)}{\partial n \partial Q} & \frac{\partial^{2} \Pi_{1}(n, Q, \xi)}{\partial n \partial \xi} \\
\frac{\partial^{2} \Pi_{1}(n, Q, \xi)}{\partial Q \partial n} & \frac{\partial^{2} \Pi_{1}(n, Q, \xi)}{\partial Q^{2}} & \frac{\partial^{2} \Pi_{1}(n, Q, \xi)}{\partial Q \partial \xi} \\
\frac{\partial^{2} \Pi_{1}(n, Q, \xi)}{\partial \xi \partial n} & \frac{\partial^{2} \Pi_{1}(n, Q, \xi)}{\partial \xi \partial Q} & \frac{\partial^{2} \Pi_{1}(n, Q, \xi)}{\partial \xi^{2}}
\end{array}\right] \cdot
$$

Therefore, we can obtain that the first-order master sequence is: 


$$
\phi_{1}=\frac{\partial^{2} \Pi_{1}(n, Q, e)}{\partial n^{2}}=s \varepsilon(a-j-k)^{2} e^{(a-j-k) n}-(c+\rho \widehat{c}) \varepsilon a^{2} e^{a n}-\frac{\varepsilon a^{2} e^{a n}}{Q}(A+\rho \widehat{A})+\rho \xi \varepsilon a^{2} e^{a n} .
$$

The second-order master sequence is

$$
\begin{aligned}
& \phi_{2}=\frac{\partial^{2} \Pi_{1}(n, Q, \xi)}{\partial n^{2}} \frac{\partial^{2} \Pi_{1}(n, Q, \xi)}{\partial Q^{2}}-\left(\frac{\partial^{2} \Pi_{1}(n, Q, \xi)}{\partial n \partial Q}\right)^{2} \\
& =-2 \varepsilon e^{a n}(A+\rho \widehat{A}) Q^{-3}\left[s \varepsilon(a-j-k)^{2} \mathrm{e}^{(a-j-k) n}-(c+\rho \widehat{c}) \varepsilon a^{2} e^{a n}+\rho \xi \varepsilon a^{2} e^{a n}\right] . \\
& +Q^{-4} \varepsilon^{2} a^{2} e^{2 a n}(A+\rho \widehat{A})^{2}
\end{aligned}
$$

The third-order master sequence is

$$
\begin{aligned}
& \phi_{3}=\frac{\partial^{2} \Pi_{1}(n, Q, \xi)}{\partial n^{2}}\left[\begin{array}{ll}
\frac{\partial^{2} \Pi_{1}(n, Q, \xi)}{\partial Q^{2}} & \frac{\partial^{2} \Pi_{1}(n, Q, \xi)}{\partial Q \partial \xi} \\
\frac{\partial^{2} \Pi_{1}(n, Q, \xi)}{\partial \xi \partial Q} & \frac{\partial^{2} \Pi_{1}(n, Q, \xi)}{\partial \xi^{2}}
\end{array}\right]-\frac{\partial^{2} \Pi_{1}(n, Q, \xi)}{\partial n \partial Q}\left[\begin{array}{ll}
\frac{\partial^{2} \Pi_{1}(n, Q, \xi)}{\partial Q \partial n} & \frac{\partial^{2} \Pi_{1}(n, Q, \xi)}{\partial Q \partial \xi} \\
\frac{\partial^{2} \Pi_{1}(n, Q, \xi)}{\partial \xi \partial n} & \frac{\partial^{2} \Pi_{1}(n, Q, \xi)}{\partial \xi^{2}}
\end{array}\right] \\
& +\frac{\partial^{2} \Pi_{1}(n, Q, \xi)}{\partial n \partial \xi}\left[\begin{array}{ll}
\frac{\partial^{2} \Pi_{1}(n, Q, \xi)}{\partial Q \partial n} & \frac{\partial^{2} \Pi_{1}(n, Q, \xi)}{\partial Q^{2}} \\
\frac{\partial^{2} \Pi_{1}(n, Q, \xi)}{\partial \xi \partial n} & \frac{\partial^{2} \Pi_{1}(n, Q, \xi)}{\partial \xi \partial Q}
\end{array}\right] \\
& =2 u \varepsilon e^{a n}(A+\rho \hat{A}) Q^{-3}\left[s \varepsilon(a-j-k)^{2} e^{(a-j-k) n}-(c+\rho \widehat{c}) \varepsilon a^{2} e^{a n}-\frac{\varepsilon a^{2} e^{a n}}{Q}(A+\rho \widehat{A})+\rho \xi \varepsilon a^{2} e^{a n}+\frac{\left(\rho \varepsilon a e^{a n}\right)^{2}}{u}\right]
\end{aligned}
$$

We can know that if

$$
\eta_{1}=s \varepsilon(a-j-k)^{2} \mathrm{e}^{(a-j-k) n}-(c+\rho \widehat{c}) \varepsilon a^{2} e^{a n}+\rho \xi \varepsilon a^{2} e^{a n}+\frac{\left(\rho \varepsilon a e^{a n}\right)^{2}}{u}<0,
$$

then $\phi_{1}<0, \phi_{1}>0$, and $\phi_{3}<0$.Therefore, the Hessian matrix is the negative definite. We can say that the unique solution $\left(n_{1}^{*}, Q_{1}^{*}, \xi_{1}^{*}\right)$ is the global maximum solution. Based on the analysis, we come up with Theorem 2.

Theorem 2. If $\eta_{1}<0$, then there exists a unique optimal solution $\left(\mathrm{n}_{1}^{*}, \mathrm{Q}_{1}^{*}, \xi_{1}^{*}\right)$ to maximize $\prod_{1}(n, Q, \xi)$.

\section{Conclusions}

Under the restriction of carbon emissions regulations, this paper investigates the trade-off between revenue and cost of trade credit considering the consumer environment sensitivity. The results can help enterprises to make reasonable credit and operational decision-making, for improving the supply chain operation performance and sustainability.

\section{Acknowledgement}

This paper is funded by the National Natural Science Foundation of China (71302115, 71502123).

\section{Reference}

Du S, Zhu L, Liang L, et al, 2013. Emission-dependent supply chain and environment-policy-making in the cap-and-trade system. Energy Policy, 57, 61-67.

Gao D, Zhao X, Geng W, 2014. A delay-in-payment contract for Pareto improvement of a supply chain with stochastic demand. Omega, 49, 60-68.

Qin J, Bai X, Xia L, 2015, Sustainable Trade Credit and Replenishment Policies under the Cap-And-Trade and Carbon Tax Regulations, Sustainability, 7(1):16340-16361. 